\title{
S4. Primary prevention of depression, neurological and psychosocial disorders
}

PRIMARY PREVENTION OF DEPRESSION

\section{R. Jenkins}

Primary prevention of depression encompasses a range of microlevel and macrolevel interventions designed to prevent the onset ofdepression. Microlevel activities may either educational or clinically focused. Macrolevel interventions are those which influence social policies. Preventive activities may be relevant for the whole population (universal strategies), a large subgroup of the population whose risk of becoming ill is above average (eg children in care) or a relatively small subgroup whose risk of becoming ill is very high (eg people who have been recently bereaved). Interventions may be both proactive (acting to reduce stressors and improve coping mechanisms) and reactive (focusing on mobilising social supports) Important places for preventive activities include schools, workplaces and primary care settings.
PRIMARY PREVENTIONOFMENTALRETARDATION.PSYCHO-SOCIAL ASPECTS

\section{Mutcahy}

Department of Psychiatry, Trinity College, Dublin 2, Ireland

The World Health Organization has a commitment to the Prevention of Mental Retardation. It has recently published guidelines in respect of specific disorders.

There are significant geographical and cultural factors to be considered in considering programmes for prevention.

The multiplicity of causes involved, new discoveries and treatment options are reviewed.

Interventions of any kind produce their own consequences - not always foreseen. Some psychosocial consequences are presented in relation to the prevention of three conditions, Phenylketonuria, Down's Syndrome and the Rh factor. 


\section{PRIMARY PREVENTION OF SUICIDE}

D. De Leo

Dept. Psychiatry, University of Padua, Via Giustiniani 2, I-35128 Padua, Italy

Suicide is among the first ten major causes of death across all age groups in most countries throughout the world. In some Westem nations it is even one of the first three causes of death in the 15-34 age group. Rates in the region of one suicide per 1,500 inhabitants are recorded in countries such as Hungary. It has been calculated that on average 400,000 people commit suicide each year throughout the world. However, there is good reason to believe that this figure may rather underestimate the phenomenon in percentages which probably range between 20 and $100 \%$. Primary prevention of suicidal behaviour implies prevention of the development of suicidal tendencies in society and in the individual. If it were really possible to prevent suicide in this way, members of society would never become suicidal. Clearly this is the most difficult method to put into practice. To be coherent, this supposes that the exact causes underlying the phenomenon of suicide are known, which is doubtful. Nonetheless, intervention can be divided up into two main forms: 1) identification of vulnerable groups; 2) restriction of access to specific suicidal methods.

Among the groups at highest risk of suicide are generally the elderly, the isolated, the depressed, unintegrated immigrants, prison inmates, etc.. Early intervention in these groups of subjects may help prevent suicide. Restriction of access to means of committing suicide may also prove to be an effective form of prevention. This includes restriction of arms ownership, detoxication of domestic gas supplies and car exhaustion gases, control of sales of toxic substances and drugs, and the "toning down" of newspaper reports concerning cases of suicide. Obviously we must also add early identification and treatment of psychiatric disorders such as depression and alcohol abuse, which are the most important risk factors for suicidal behaviour.

\section{PRIMARY PREVENTION OF EPILEPSY}

H. Meinardi

Institute of Neurology, Department of Epileptology, Catholic University, c/o Postbus 21, 2100 AA Heemstede, The Netherlands.

Primary prevention of epilepsy may appear wishful thinking if one considers that $65 \%$ of seizures are idiopathic and only $35 \%$ remote symptomatic. Primary prevention of the consequences of epilepsy are much more important. In fact one should keep in mind that epilepsy is a syndrome of which the main features are associated with paroxysmal excessive discharges of nerve cells but which has concomitant features elicited because seizures occur. The psychological consequences of suffering from epilepsy should be preventable though few systematic studies have been undertaken to verify this hypothesis. The impact of chronic antiepileptic drug use is amenable to primary prevention by careful selection of the drug to be used and by therapeutic monitoring. Therapeutic monitoring should not only concern itself with appropriate assessment of serum levels of the drug, it should also involve rational use of polytherapy if indicated and quantitative registration of side effects. In developing countries primary prevention of the consequences of epilepsy has to include case-finding, public education and improved drug procuration. 